\title{
Cryobiopsy versus forceps biopsy in endobronchial lesions, diagnostic yield and safety
}

The authors declare no financial disclosure

\begin{abstract}
Introduction: This study aimed to evaluate the safety and diagnostic yield of cryobiopsy (CB) in comparison to forceps biopsy in endobronchial lesions.

Material and methods: Patients with suspected endobronchial lesions were enrolled. Two forceps biopsies and one cryobiopsy were done in the same patient with randomized sequence. The largest diameter of the samples was measured in mm by electronic caliper. Diagnostic yield of each technique and postbronchoscopy bleeding were evaluated.

Results: Samples obtained by CB was significantly larger than that of the forceps biopsy $(5.9 \pm 2.3$ vs $2.5 \pm 0.8, p=0.001)$. Diagnostic yield of CB was significantly higher than forceps biopsy $74.5 \%$ versus $51.1 \%(p=0.001)$. Mild and moderate bleeding grades were reported in both techniques with no significant difference $(p=0.063, p=0.5)$, respectively. Severe bleeding was not recorded in both techniques.

Conclusions: $\mathrm{CB}$ represents a safe and effective tool to obtain a larger tissue samples of a good quality with higher diagnostic yield in comparison to standard forceps samples. On the other hand, bleeding occurred more frequently after CB than forceps biopsy. However, without severe adverse effects.
\end{abstract}

Key words: cryobiopsy, forceps biopsy, lung cancer, bronchoscopy, endobronchial lesion

Adv. Respir. Med. 2017; 85: 301-306

\section{Introduction}

One of the main goals of diagnostic bronchoscopy, besides visualization of endobronchial abnormalities, is obtaining an adequate tissue samples from the suspicious lesions for cytohistological examination. Several techniques could be applied through the working channel of the flexible bronchoscope (FB), such as forceps biopsy, brush, bronchial washing and transbronchial needle aspiration (TBNA) [1].

Forceps biopsy is the standard method to obtain tissue samples from airway lesions, with a diagnostic yield ranging between $65-82 \%[2,3]$. The main drawback of forceps biopsy is the small sized tissue samples. Its diameter is about $2 \mathrm{~mm}$. Additionally, uncertain representation of the tumor tissue may occur due to presence of tumor heterogeneity $[4,5]$. Larger samples are essential for definite pathological diagnosis, as well as immunohistochemical staining and detection of target specific genetic alterations in the tumor tissue, which has an increasing importance in the treatment of lung cancer [6]. Forceps biopsy samples are often associated with mechanical damage and crushing, which may cause difficulties in interpretation of anatomic structure and histopathologic changes in the specimens.

Flexible cryoprobes were introduced as a new tool for bronchoscopic tissue sampling. It is used

Address for correspondence: Ahmed Ehab, Chest Medicine Department, Faculty of Medicine, Mansoura University, Elgmoheria Str., 35151, Mansoura, Egipt DOI: 10.5603/ARM.2017.0052

Received: 26.09.2017

Copyright (C) 2017 PTChP

ISSN 2451-4934 
primarily for debulking and cryoextraction of malignant airway stenosis $[7,8]$. With this technique, the sample is collected while still being frozen, with the tissue attached on the frozen tip of the probe. By this way, larger tissue samples can be taken from endobronchial lesions. Tissue samples obtained with cryoprobes are of good quality, size and artifact-free. The molecular markers are also better preserved and well represented [9].

The aim of our study was to compare bronchoscopic cryobiopsy versus forceps biopsy in patients with suspected endobronchial lesions as regards the size and diagnostic yield of the samples and safety of the procedure.

\section{Material and methods}

This is a prospective, randomized controlled study conducted at Chest Medicine Department - Mansoura University, Egypt from January 2014 to May 2016.

Patients with clinically and radiologically suspected endobronchial lesions and planned for diagnostic bronchoscopy and biopsy taking were enrolled in this study, after approval of the ethical committee of Mansoura Faculty of Medicine (code number MS/243). Before FB, a clear explanation of the procedure to the patient was done and fully detailed consent was signed by the patient.

\section{Inclusion criteria}

All patients must be fit for FB, with suspected endobronchial lesions and with normal coagulation profile.

\section{Exclusion criteria}

Patients, who refused to be included in this study or unfit for FB were excluded from the study or with uncorrectable bleeding profile. All patients were subjected to: medical history and clinical examination, laboratory investigations as complete blood count, serum creatinine, liver enzymes, international normalized ratio (INR), radiological assessment and arterial blood gases (ABG) at room air.

\section{The procedure:}

1. Bronchoscopic intervention:

FB was done using (PENTAX FB-18V fiber bronchoscopy or PENTAX EB-1970TK Video bronchoscopy) after instillation of lidocaine $2 \%$ and IV sedation of midazolam. Continuous monitoring for $\mathrm{O}_{2}$ saturation and ECG throughout and within
2 hours after the procedure were conducted. All procedures were done without intubation of the patients. Both forceps and cryobiopsy were performed in the same patient. The sequence of the biopsies was randomized with a closed envelope method. The number of biopsies were 2 biopsies for the forceps versus one cryobiopsy.

The cryoprobe used in this study was flexible cryorecanalization probe $(78 \mathrm{~cm}$ in length $/ 2.4 \mathrm{~mm}$ in diameter, ERBE, Medizintechnik GmbH, Tübingen, Germany) which was connected to cryomachine (ERBOCRYO CA, ERBE, Tübingen, Germany), while the forceps used in the study was (Endo-Flex biopsy forceps $2.3 \mathrm{~mm}$ diameter and $180 \mathrm{~cm}$ length, Voerde, Germany).

2. Tissue sampling:

Bronchoscopic forceps biopsy was done as usual from the visualized endobronchial lesions according to Gasparini [10]. Two forceps biopsies were taken per patient, after the second biopsy the amount of bleeding was evaluated, categorized and evaluation of the methods used to control it.

The cryobiopsy samples were obtained by advancement of the cryoprobe into the working channel of the bronchoscope to touch the tip of the endobronchial tumor. The freezing time was approximately 4 seconds. Then, FB together with tissue sample attached to the tip of the frozen probe were extracted en toto outside the bronchial tree. The tissue sample was released from the probe's tip by plunging it into saline at room temperature. FB was reintroduced after cryobiopsy to evaluate and control the bleeding.

3. Intervention evaluation:

The following were evaluated and recorded:

I. The sum size of the forceps biopsy samples and size of cryobiopsy were measured by an electronic caliper in its largest diameter in mm (Fig. 1).

II. Post interventional bleeding: according to Aktas et al. [11], bleeding was classified into:

- No bleeding.

- Mild bleeding, which was controlled by maintained suction.

- Moderate bleeding, which can be controlled with different interventional techniques e.g local application of saline either normal or cold, adrenalin or balloon tamponade.

- Severe bleeding, which necessitates ICU admission due to hemodynamic instability and required blood transfusion.

4. Histopathological evaluation:

Specimens were fixed in formalin $10 \%$, and sent for pathological examination. The diagnostic 


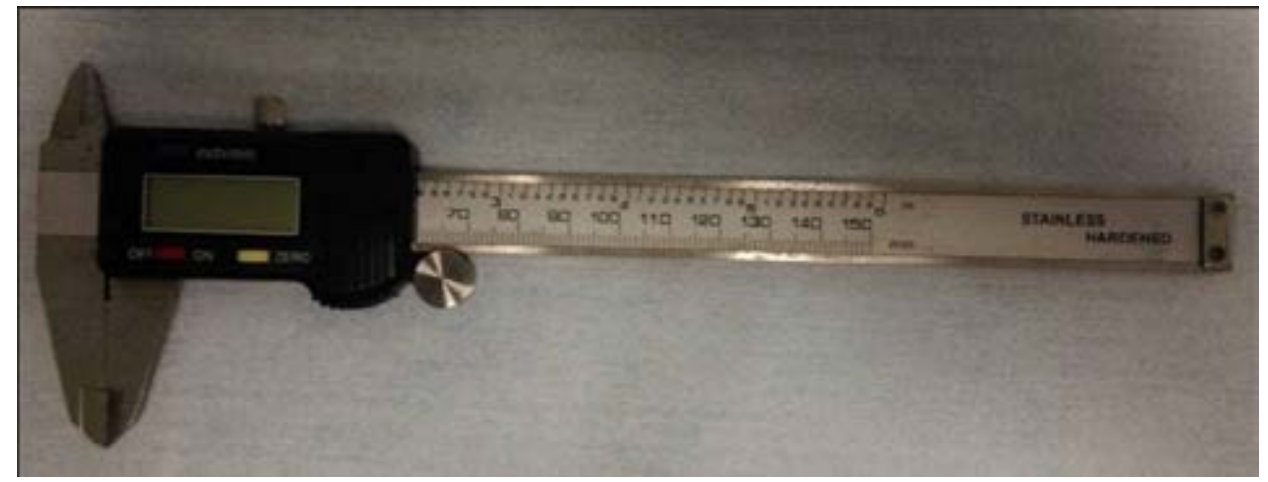

Figure 1. Electronic caliper device used in the measurement of the tissue samples

yield of each technique was demonstrated. A diagnostic technique was only defined when a final histopatholocal diagnosis was obtained.

5. Statistical analysis:

Categorical data were presented as number and percentage. Kolmogorov-Smirnov test was used to check data normality. Normally distributed data were presented as mean \pm standard deviation (SD); paired sample t-test was used to compare the results in the same group. Non-normally distributed data were presented as median. Non-parametric two-related-samples test (Wilcoxon type) was used to compare the results in the same group. McNemar test was used to compare paired proportions. $\mathrm{P}<0.05$ was considered as statistically significant. Statistical analysis was performed using Excel and SPSS software (v.23).

\section{Results}

This study included 47 patients, 32 males (68.1\%) and 15 females (31.9\%) with a mean age of $55.47 \pm 11.57$. Twenty three patients (49\%) were non-smokers and 21 patients (44.68\%) were current smokers and 3 patients (6.38\%) were exsmokers as shown in Table 1.

The total number of biopsies was 94 forceps versus 47 cryobiopsies. The distribution of biopsies sites was illustrated in Table 2. Biopsy size; cryobiopsies were significantly larger than forceps biopsies with a mean size of $5.9 \pm 2.3$ and $2.5 \pm 0.8 \mathrm{~mm}$ respectively ( $\mathrm{p} \leq 0.001)$ (Fig. 2).

The final diagnosis was established in 35 patients $(74.5 \%)$ with cryobiopsy versus 24 patients (51.1\%) with forceps biopsy, the diagnostic yield of the cryobiopsies were statistically significantly higher than forceps biopsies $(p=0.001)$. The final diagnosis is listed in Table 3.

No bleeding was associated with forceps biopsy in $74.5 \%$ of cases versus $59.6 \%$, this

\section{Table 1. Demographic data of the studied patients}

\begin{tabular}{lc}
\hline \multicolumn{2}{c}{ Total number of the patients: $\mathbf{4 7}(\mathbf{1 0 0 \% )}$} \\
\hline Age: (mean \pm SD) & $\mathbf{5 5 . 4 7} \pm \mathbf{1 1 . 5 7}$ \\
\hline Sex & \\
Male & $32(68.1 \%)$ \\
Female & $15(31.9 \%)$ \\
Smoking status & \\
Non smoker & $23(49 \%)$ \\
Current smoker & $21(44.7 \%)$ \\
Ex-smoker & $3(6.4 \%)$ \\
\hline
\end{tabular}

difference was significant $(p=0.016)$. Mild and moderate bleeding grades were reported in both techniques with no significant difference $(\mathrm{p}=$ 0.063 and $p=0.5$ ), respectively. Severe bleeding was not recorded in both techniques (Table 4).

There was no reported severe bleeding after both techniques as well as no reported other complications including pneumothorax, pneumomediastinum or mortality.

\section{Discussion}

Forceps biopsy is the traditional method for diagnosis of bronchoscopically visible lesions [12]. Its diagnostic yield is affected by diameter, location, pathologic nature, visibility of the tumor and importantly the size and quality of the obtained samples [11, 13].

The diagnostic yield of forceps biopsy can be augmented with the use of additional tools as brushings, needle aspirates, and washings. However, the overall cost, time and possible complications including anesthesia will be increased [12].

The relatively small size of the biopsies, crushing effect and low diagnostic value of the 
Table 2. Sequence, number, site and size and diagnostic yield of bronchoscopic biopsies taken from the studied patients

\begin{tabular}{|c|c|c|c|}
\hline \multicolumn{4}{|c|}{ Total number of the patients: $47(100 \%)$} \\
\hline & Forceps & Cryobiopsy & $\mathbf{p}$ \\
\hline Sequence of biopsies & $\begin{array}{l}\text { Forceps then cryo } \\
23(48.9 \%)\end{array}$ & $\begin{array}{c}\text { Cryo then forceps } \\
24(51.1 \%)\end{array}$ & 0.5 \\
\hline \multicolumn{4}{|l|}{ Number of the biopsies } \\
\hline - Total biopsies number & 94 & 47 & - \\
\hline - Biopsies per patient & 2 & 1 & $\leq 0.001$ \\
\hline \multicolumn{4}{|l|}{ Distribution of the biopsy site: } \\
\hline - Rt main bronchus & 8 & 4 & \\
\hline - Rt upper lobe & 6 & 3 & \\
\hline - Bronchus intermedius & 14 & 7 & \\
\hline - Middle lobe & 8 & 4 & _- \\
\hline - Rt lower lobe bronchus & 4 & 2 & \\
\hline - Let main bronchus & 18 & 9 & \\
\hline - Lt upper lobe & 18 & 9 & \\
\hline - Lingula & 4 & 2 & \\
\hline - Lt lower lobe & 14 & 7 & \\
\hline Size of the biopsy in mm (mean \pm SD) & $2.5 \pm 0.8$ & $5.9 \pm 2.3$ & $\leq 0.001$ \\
\hline Diagnostic yield & $24(51.1 \%)$ & $35(74.5 \%)$ & 0.001 \\
\hline
\end{tabular}

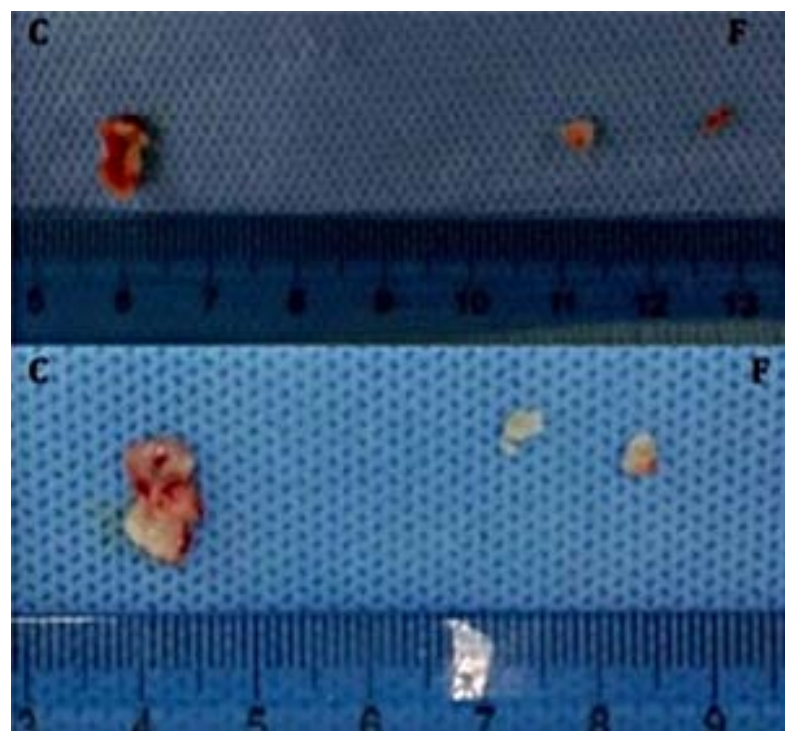

Figure 2. Tissue samples; $\mathrm{C}$ - cryobiopsy; F - forceps biopsy

forceps are a motive to apply the cryobiosy as a new biopsy tool. Cryobiopsy samples have a good quality with a higher diagnostic yield in comparison to forceps biopsy. The cryobiopsy samples are significantly larger than samples obtained by forceps samples [11].

Flexible cryoprobe and cryomachine used in our department has the largest commercial-
Table 3. Final diagnosis of the studied patients

\begin{tabular}{lc}
\hline \multicolumn{2}{c}{ Total number of the patients: $\mathbf{4 7} \mathbf{( 1 0 0 \% )}$} \\
\hline Diagnosis & $\mathbf{n}(\%)$ \\
\hline Chronic suppurative inflammation & $2(4.3)$ \\
Bengin polyps & $2(4.3)$ \\
Pulmonary hamartoma & $1(2.1)$ \\
Adenocarcinoma & $13(27.7)$ \\
Small cell lung cancer & $5(10.6)$ \\
Squamous cell carcinoma & $5(10.6)$ \\
Large cell carcinoma & $4(8.5)$ \\
Small cell Lymphoma & $1(2.1)$ \\
Pleomorphic carcinoma & $1(2.1)$ \\
Papillary carcinoma & $1(2.1)$ \\
Carcinoid tumor & $1(2.1)$ \\
Spindle cell tumor & $1(2.1)$ \\
No final diagnosis & $10(21.3)$ \\
\hline
\end{tabular}

ly available diameter. It allows to obtain larger samples than the other probes $(1.9 \mathrm{~mm})$. In our study, contact time between the cryoprobe and endobronchal lesion was approximately 4 seconds. By increasing contact time, the size of the obtained samples enlarges. This may be explained 
Table 4. Bleeding complication after forceps and cryobiopsy

\begin{tabular}{lccc}
\hline & \multicolumn{4}{l}{ Total number of the patients: $\mathbf{4 7}$ (100\%) } & p \\
\hline & Forceps $\mathbf{n}(\%)$ & Cryobiopsy $\mathbf{n}(\%)$ & 0.016 \\
\hline No bleeding & $35(74.5)$ & $28(59.6)$ & \\
Bleeding & & & 0.063 \\
• Mild & $10(21.3)$ & $15(31.9)$ & 0.5 \\
•Moderate & $2(4.3)$ & $4(8.5)$ & \\
\hline
\end{tabular}

by concentric expansion of the freezing area starting from the tip of the cryoprobe and expanding into the periphery, making a larger surface area and thereby larger biopsies can be generated [6]. The used forceps in our study was chosen based on its larger diameter which can obtain larger tissue samples.

It was found that the mean size of the crobiopsy samples was $5.9 \pm 2.3 \mathrm{~mm}$ while for forceps biopsy $-2.5 \pm 0.8 \mathrm{~mm}$. With contact time 4 seconds, the size of the cryobiopsy was almost 2 folds larger than those obtained by the forceps biopsy. We have obtained tissue samples up to $16.70 \mathrm{~mm}$ by the cryobiopsy technique.

Our study was comparable to the study done by Aktas et al. [11], in which median size of cryobiopsy and forceps samples were $0.8 \mathrm{~cm}$ $(0.3-4.0 \mathrm{~cm})$ and $0.2 \mathrm{~cm}(0.1-1.0 \mathrm{~cm})$, respectively $(\mathrm{p}<0.001)$.

Jabari et al. [14] investigated both forceps and cryobiopsy in 60 patients with endobronchial lesion. Forceps biopsy and cryobiopsy in three and five seconds were done. They concluded that specimens that were obtained by cryobiopsy in five seconds were significantly larger than those of forceps biopsy and cryobiopsy in three seconds $(\mathrm{p}<0.001)$. We showed that the achieved diagnostic yields for all three methods were not statistically different $(p>0.05)$. Simultaneous usage of samples produced in both cryobiopsies can significantly improve the diagnostic yield $(p=0.02)$.

In our study, the diagnostic yield of single cryobiopsy was $74.5 \%$ while the double foreceps biopsy was $51.1 \%$. This is explained by both high quality and larger size of the cryobiopsy samples. Also, the cryobiopsy can establish the final diagnosis without the need of additional interventions or the need of repeated bronchoscopy or referring the patients for another diagnostic surgical intervention.

The diagnostic yield of cryobiopsy in our study $(74.5 \%)$ is less than that reported by Hetzel et al. [6] (95\%) and the diagnostic yield of double forceps biopsy in our study (51.1\%) is less than their reported results of forceps (85.1\%). The cause of this difference may be attributed to higher number included in the study compared to our study. They included a total number of 563 divided into 2 groups; 281 patients were subjected to forceps biopsies and 282 subjected to cryobiopsies. In addition, the number of the biopsy in the above mentioned study was left to the investigator, while in our study we have a fixed number of the biopsies in the form of 2 forceps biopsies and one cryobiopsy

Also, Aktas et al. [11] illustrated that the diagnostic yield of the cryobiopsy was higher than the forceps biopsy, they investigated both cryobiopsy and forceps biopsy in 41 patients with endobronchial lesions. Both techniques were done in each patient with 3 forceps biopsies and one cryobiopsy. The final diagnosis was obtained in 38 patients ( $92.7 \%)$ versus $32(78 \%)$ in forceps biopsy. This difference may be related to different number of the examined patients in both studies.

Schumann et al. [7] examined a total number of 296 patients with visible endoluminal tumor. The final included group consisted of 55 patients, where both cryobiopsy and forceps biopies were done in the same patient. A significantly higher diagnostic yield of cryobiopsy compared with forceps biopsy $(89.1 \%$ vs $65.5 \%, p<0.05)$ was observed.

The safety of the procedure was also another important aspect in our study. Forceps biopsy was associated with no bleeding in 35 (74.5\%) and cryobiopsy in 28 (59.6\%), with significant difference between both $(\mathrm{p}=0.016)$.

As regards bleeding, cryobiopsy was associated with more bleeding (mild: 15 [31.9\%] and moderate 4 [8.5\%]) in comparison to forceps biopsy (mild 10 [21.3\%] and moderate 2 [4.3 $\%]$ ) without any significant difference. No severe bleeding was reported after both techniques. However, as expected no bleeding was more frequently observed after forceps biopsy. Mild and moderate bleeding was observed more often after cryobiopsy. The bleeding could be control- 
led with the usual interventions including: cold saline, mentioned suction or local application of adrenalin.

High incidence of bleeding during cryobiopsy would be possibly caused by extraction of the cryoprobe, when a tissue sample may attach to the end of the probe or endoscope. This may extend the time without local control of bleeding and accumulation of blood at the biopsy site. Such situations were not observed in the animal study [6].

Our results were similar to the study of Hetzel et al. [6], who reported significantly more bleeding in the cryobiopsy group in comparison to the forceps group $(p=0.009)$. However, the number of bleeding that needed any intervention for haemostasis control did not differ between the groups $(p=0.9)$. Argon plasma coagulation was required in 13 patients from the cryobiopsy group compared with eight patients in the forceps group. Tamponade was required in two patients in each group. All other episodes of bleeding were managed by instillation of ice-cold saline or vasoconstrictive drugs. No surgical interventions for bleeding control were needed and no fatal events occurred.

Aktas et al. [11] investigated the safety of both cryobiopsy and forceps biopsy in 41 patients with endobronchial lesions. There was no significant difference between these two procedures in the incidence of hemorrhage ( $p>0.05)$. This was found that $34.1 \%$ and $36.6 \%$ of hemorrhage occurred following forceps and cryoprobe biopsies, respectively. The difference between forceps biopsies and cryoprobe biopsies in terms of complication was not statistically significant ( $\mathrm{p}>0.05)$.

One of the main limitation of the cryobiopsy was the need of endotracheal intubation during the intervention to secure the airways and allow rapid reintroduction of the bronchoscope as the tissue samples obtained by cryobiopsy cannot be extracted through the working channel of the bronchoscope. In our study, none of the examined patients were intubated. We rapidly reintroduced the bronchoscope immediately after the biopsy. No additional adverse events were observed. Although intubation using flexible endotracheal tube or rigid bronchoscopy is generally recommended [6].
Despite the relative small number of patients in our study, it suggests the superiority, and will contribute to growing evidence supporting the use of bronchoscopy cryobiopsy in the diagnosis of endobronchial lesions.

\section{Conflict of interest}

The authors declare no conflict of interest.

\section{References:}

1. Dionísio J. Diagnostic flexible bronchoscopy and accessory techniques. Rev Port Pneumol. 2012; 18(2): 99-106, doi: 10.1016/j.rppneu.2012.01.003, indexed in Pubmed: 22317896.

2. Rivera MP, Mehta AC. American College of Chest Physicians. Initial diagnosis of lung cancer: ACCP evidence-based clinical practice guidelines (2nd edition). Chest. 2007; 132(3 Suppl): 131S-148S, doi: 10.1378/chest.07-1357, indexed in Pubmed: 17873165.

3. Rivera MP, Detterbeck F, Mehta AC, et al. American College of Chest Physicians. Diagnosis of lung cancer: the guidelines. Chest. 2003; 123(1 Suppl): 129S-136S, indexed in Pubmed: 12527572.

4. Aleva RM, Kraan J, Smith M, et al. Techniques in human airway inflammation: quantity and morphology of bronchial biopsy specimens taken by forceps of three sizes. Chest. 1998; 113(1): 182-185, indexed in Pubmed: 9440588.

5. Griff S, Ammenwerth W, Schönfeld N, et al. Morphometrical analysis of transbronchial cryobiopsies. Diagn Pathol. 2011; 6: 53, doi: 10.1186/1746-1596-6-53, indexed in Pubmed: 21679402 .

6. Hetzel J, Eberhardt R, Herth FJF, et al. Cryobiopsy increases the diagnostic yield of endobronchial biopsy: a multicentre trial. Eur Respir J. 2012; 39(3): 685-690, doi: 10.1183/09031936.00033011, indexed in Pubmed: 21852332.

7. Schumann C, Hetzel J, Babiak AJ, et al. Cryoprobe biopsy increases the diagnostic yield in endobronchial tumor lesions. J Thorac Cardiovasc Surg. 2010; 140(2): 417-421, doi: 10.1016/j. jtcvs.2009.12.028, indexed in Pubmed: 20226474.

8. Hetzel M, Hetzel J, Schumann C, et al. Cryorecanalization: a new approach for the immediate management of acute airway obstruction. J Thorac Cardiovasc Surg. 2004; 127(5): 1427-1431, doi: 10.1016/j.jtcvs.2003.12.032, indexed in Pubmed: 15116003 .

9. Babiak A, Hetzel J, Krishna G, et al. Transbronchial cryobiopsy: a new tool for lung biopsies. Respiration. 2009; 78(2): 203208, doi: 10.1159/000203987, indexed in Pubmed: 19246874.

10. Gasparini S. Conventional Biopsy Techniques. Principles and Practice of Interventional Pulmonology. 2012: 151-163, doi: 10.1007/978-1-4614-4292-9 15.

11. Aktas Z, Gunay E, Hoca NT, et al. Endobronchial cryobiopsy or forceps biopsy for lung cancer diagnosis. Ann Thorac Med. 2010; 5(4): 242-246, doi: 10.4103/1817-1737.69117, indexed in Pubmed: 20981186.

12. Rubio ER, le SR, Whatley RE, et al. Cryobiopsy: should this be used in place of endobronchial forceps biopsies? Biomed Res Int. 2013; 2013: 730574, doi: 10.1155/2013/730574, indexed in Pubmed: 24066296.

13. Roth K, Hardie JA, Andreassen AH, et al. Predictors of diagnostic yield in bronchoscopy: a retrospective cohort study comparing different combinations of sampling techniques. BMC Pulm Med. 2008; 8: 2, doi: 10.1186/1471-2466-8-2, indexed in Pubmed: 18221551.

14. Jabari H, Sami R, Fakhri M, et al. Different protocols for cryobiopsy versus forceps biopsy in diagnosis of patients with endobronchial tumors. Pneumologia (Bucharest, Romania). 2011 Dec. ; 61(4): 230-233. 